\title{
Henoch-Schönlein purpura
}

\author{
Danny Guo MD MSc, Joseph M. Lam MD
}

A previously healthy 12-year-old girl presented to our outpatient pediatric dermatology clinic with a three-week history of an itchy, red raised rash on her lower extremities that had spread to her buttocks. She also reported abdominal pain, swollen feet and joint pain but could not recall seeing blood in her urine. Upon examination, the patient had palpable purpuric plaques over her lower extremities (Figure 1) with mild periarticular swelling of her ankles. A urinalysis showed microscopic hematuria, whereas results from blood tests, including renal function and complete blood cell count, were normal. We diagnosed Henoch-Schönlein purpura, and the patient and her parents were educated about the syndrome. She had complete resolution of skin lesions and microhematuria after four months. She did not require any medications and was followed-up with monthly urinalysis tests.

Henoch-Schönlein purpura is a vasculitis mediated by immunoglobulin A (IgA), with an incidence of about 22 cases per 100000 per year. ${ }^{1}$ It is slightly more common in children, white people and men. ${ }^{1}$ It is characterized by palpable purpuric papules arising symmetrically on dependent areas of the body. About $50 \%$ of patients with Henoch-Schönlein purpura have renal involvement, but less than $1 \%$ of these patients progress to end-stage renal failure. ${ }^{2}$ Vasculitis can affect the joints, gastrointestinal tract, lungs, scrotum and, uncommonly, the central nervous system.

A diagnosis of Henoch-Schönlein purpura should be suspected in children with abdominal pain, arthritis, renal impairment and nonthrombocytopenic purpura. Other diagnoses to consider include drug reactions, idiopathic thrombocytopenic purpura, Rocky Mountain spotted fever, disseminated gonococcemia and urticarial vasculitis. ${ }^{1}$ Biopsies of the skin are not usually performed but generally show leukocytoclastic vasculitis and IgA deposits. Renal biopsies may be indicated if renal dysfunction persists or worsens. ${ }^{1}$

Treatment of Henoch-Schönlein purpura is mostly supportive and includes patient education and symptom control with nonsteroidal anti-

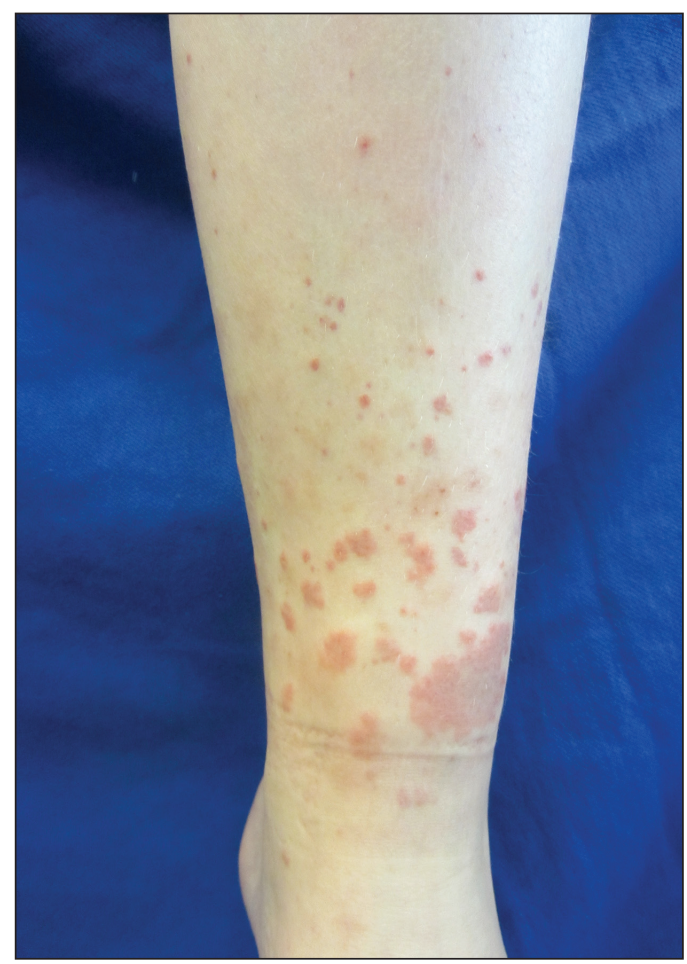

Competing interests: None declared.

This article has been peer reviewed.

The authors have obtained patient consent.

Affiliations: Department of Dermatology and Skin Science (Guo), University of British Columbia; Departments of Pediatrics and Dermatology (Lam), University of British Columbia, Vancouver, BC.

Correspondence to: Joseph Lam, joseph.mc. lam@gmail.com

CMAJ 2016. DOI:10.1503 /cmaj.151072
Figure 1: Palpable purpuric and ecchymotic plaques on the left leg of a 12-year-old girl.

inflammatory drugs. ${ }^{2}$ Systemic prednisone can alleviate severe joint and abdominal pain, but it does not prevent renal disease. ${ }^{3}$ In patients with normal results on initial urinalysis tests or with isolated hematuria, a monthly urinalysis should be performed for six months to detect renal involvement. ${ }^{2,3}$ Admission to hospital should be considered if substantial renal compromise or gastrointestinal bleeding occur.

\section{References}

1. Reamy BV, Williams PM, Lindsay TJ. Henoch-Schönlein purpura. Am Fam Physician 2009;80:697-704.

2. Chen JY, Mao JH. Henoch-Schönlein purpura nephritis in children: incidence, pathogenesis and management. World $J$ Pediatr 2015;11:29-34.

3. Hahn D, Hodson EM, Willis NS, et al. Interventions for preventing and treating kidney disease in Henoch-Schönlein purpura (HSP). Cochrane Database Syst Rev 2015;8:CD005128. 